\title{
An Efficient Technique for Dynamically Allocating Channels in Satellite Cellular Networks
}

\author{
Enrico Del Re, Romano Fantacci, Giovanni Giambene \\ Dipartimento di Ingegneria Elettronica - Università di Firenze \\ Via S. Marta, 3 - 50139 Firenze, Italy \\ Tel. +39554796270 - Fax +39554796485 - Telex 580681 UNIFING-I
}

\begin{abstract}
This paper proposes an improved technique for dynamically allocating channels in mobile satellite cellular networks. Channel assignment on demand is performed on the basis of the evaluation of a cost-function. When the cell of a new call arrival has not available channels, a single channel reconfiguration in an interfering cell is attempted in order to accept a new call. Handover requests that do not receive immediate service can be queued for a maximum time. The scenario envisaged is Low Earth Orbit - Mobile Satellite Systems (LEO-MSSS). A performance comparison with other dynamic channel allocations has been carried our in order to hightight the higher efficiency of the dynamic technique proposed.
\end{abstract}

\section{INTRODUCTION}

The steady growth in the cellular telephony market requires an ever increasing capacity in order to meet the range of services and the foreseen wide population of users of the future "Personal Communication Services" (PCS) [1],[2]. PCS will encompass, in a unique standard, the different technologies of cordless, terrestrial and satellite mobile cellular networks. At present, R\&D efforts are addressed towards the definition of Mobile Satellite Systems (MSSs) that use a constellation of NonGeoStationary Orbit (NGSO) satellites. In particular, Low Earth Orbit satellites (LEO-MSSs) are considered since they permit to relax the constraints on the budget link and allow the use of lowpower hand-held mobile terminals. Several LEO-MSSs have been proposed, e.g., IRIDIUM (by Motorola), ARIES (by Constellation Communications Inc.), GLOBALSTAR (by Loral Qualcomm Satellite Services Inc.), TELEDESIC (the former CALLING, by Microsoft et al.). The working example assumed in this paper is given by the IRIDIUM system [3], which is where $T_{n}$ represents the average call duration.

formed by 66 satellites over 6 polar circular orbits at about 780 Parameters $R$ and $V_{r r k}$ depend on the satellite constellation $\mathrm{km}$ of altitude. Each satellite covers a 48-cell-network with a altitude; moreover, $R$ is also dependent on the Half Power Beam multi-spot-beam antenna. However, the techniques described here Width of the spot-beams from the satellite antenna. By can be directly. applied also to other satellite orbital considering a fixed value for $T_{m}$, the MS mobility increases if $V_{r r k}$ configurations. The inter-beam handover request procedure is increases and/or $R$ decreases (i.e., $\alpha$ decreases). In the IRIDIUM assumed based on the signal quality received by the mobile user. case, it has been considered $R=212.5 \mathrm{~km}$ and $V_{t r k} \approx 26,600$ Accordingly to a de-centralized implementation, the handover $\mathrm{km} / \mathrm{h}$. Further details about this mobility model are given in [5]. procedure is "mobile controlled": each active Mobile Station (MS) continuously checks the power level, $p$, received from the spot-beam managing it and compares this level with that relative III. to other "visible" spot-beams, $p_{\mathrm{i}}$. A handover request is issued

by the MS to the satellite (currently managing it) whenever $p<$ $p_{i}-h$ ( $h=$ hysteresis level) and $p$ falls below a given threshold, $s$ [4]. If a handover procedure cannot be completed in the destination cell of the mobile user the communication is dropped and lost. In this paper, an efficient Dynamic Channel Allocation (DCA) technique is proposed which achieves a performance improvement by allowing a channel rearrangement at the call arrival instant. Channel allocation performance has been derived in terms of probability that a call is not completely served, $P_{n s}$, owing to the initial blocking or the failure of a subsequent handover procedure (=call dropping). It will be shown that the DCA technique proposed attains the lowest $P_{n s}$ values with respect to other approaches previously proposed in the literature.

\section{A MOBILITY MODEL}

The coverage area has been assumed divided into cells and each cell is illuminated by an antenna spot-beam from a satellite. All the cells are disposed according to a hexagonal regular layout nd have a circular shape obtained by means of beam-forming in to compensate the footprint distortion due to the spherical the earth surface. It has been shown [5] that a (n) LEO mobility scenario is that of antenna beams on the earth with a speed equal to the ground-track speed of satellites, $V_{r r k}$. The user mobility will be characterized in what follows by the parameter $\alpha$, defined as:

$$
\alpha \triangleq \frac{2 R}{V_{t r k} T_{m}}
$$

\section{A DYNAMIC CHANNEL ALLOCATION TECHNIQUE FOR LEO-MSSS}

In this section, a DCA technique is proposed to allocate Work carried out under the financial support of MURST and ASI. resources efficiently. Propagation and interference 
considerations were simply represented by the constraint that, if a channel is used in a given cell $x$, it cannot be re-used in two tiers of cells around that cell. These cells form the belt of interfering cells of $x, l(x)$. Accordingly, the minimum distance for two co-channel cells, $D$, (i.e., the "reuse distance") has been set. Let us denote with $\Lambda(x)$, the set of the channels not used in $x$ and in $I(x)$.

\section{A) Management of new call attempts}

Let us assume that a new call arrival must be served in cell $x$ :

1) if it results $\Lambda(x) \neq \varnothing$, the best channel $i^{*}$ to be allocated is selected according to the following rule:

$$
C_{x}\left(i^{*}\right)=\min _{l \in \Lambda(x)}\left\{C_{x}(i)\right\}
$$

Terms $C_{x}(i)$ in (2) denote the cost-function per channel $i$. This function will be defined later in this Section.

If more channels verify (2), a random choice is performed.

The channel allocation technique previously proposed in [5], [6] blocks the new call arrival if $\Lambda(x)=\varnothing$; conversely, the DCA technique under consideration permits to serve new call arrivals by means of the "Persistent Polite Aggressive" (PPA) approach [7] even if $\Lambda(x)=\varnothing$, as outlined below.

2 If it results $\Lambda(x)=\varnothing$, the PPA mechanism defines the elements (if any) of the set of couples, $\Omega(x)=\{(\beta, \delta)\}$, where the generic couple $(\beta, \delta)$ represents a channel $\beta$ that results locked in cell $x$ only by its use in cell $\delta \in I(x)$. Then, within $\Omega(x)$, we must exclude the couples $(\beta, \delta)$, where cell $\delta$ has no available channel:

$$
\Gamma(x)=\{(\beta, \delta) \in \Omega(x): \Lambda(\delta) \neq \emptyset\}
$$

- If $\Gamma(x) \neq \varnothing$, each couple $(\beta, \delta) \in \Gamma(x)$ represents a possible channel rearrangement solution in an interfering cell of $x$ to serve the new call arrival in $x$. Then, the most convenient channel rearrangement, characterized by the couple $\left(\beta^{*}, \delta^{*}\right)$, is performed:

$$
\delta^{*}: Z\left\{\Lambda\left(\delta^{*}\right)\right\}=\max _{\Gamma(x)}\{Z\{\Lambda(\delta)\}\}
$$

where $Z\{\theta\}$ gives the number of elements belonging to the discrete set $\theta$.

In cell $\delta^{*}$, channel $\beta^{*}$ is de-allocated and the call in progress on $\beta^{*}$ is passed on another channel according to the assignment criterion outlined in the first step of this algorithm. Then, channel $\beta^{*}$ becomes available in cell $x$ and it is used to serve new call arrivals.

- If $\Gamma(x)=\varnothing$, the call attempt in $x$ is blocked and lost.

This algorithm is "polite-aggressive" since it tries to serve a new call arrival (otherwise blocked) by allowing a channel rearrangement in an interfering cell. Moreover, this technique is also "persistent" because it tries to obtain this result by considering each possibility given by the set $\Omega(x)$, without limiting itself to only one or two attempts. In the following, it will be proven that the complexity introduced in the allocation algorithm by the second step is negligible and can be supported by the system.

The cost-function $C_{x}(i)$ used in (2) is described below. We start by considering the channels allocated according to a Fixed Channel Allocation (FCA) [5], assuming a reuse distance equal to $D$. Let $F_{D}(x)$ be the set of channels assigned to cell $x$ according to FCA. A fixed allocation assures a distribution of channels among the cells of the network, with the minimum possible reuse distance, $D$. With the proposed technique, we select channels belonging to $F_{D}(x)$, whenever possible, in allocating channels to cell $x$. The allocation cost contribution for channel $i \in \Lambda(x)$, due to the interfering cell $k \in I(x), C_{x}(k, i)$, can be expressed as:

$$
C_{x}(k, i)=u_{k}(i)+2\left(1-q_{k}(i)\right), \forall k \in I(x)
$$

Where $u_{k}(i)$ and $q_{k}(i)$ are given by:

$$
\begin{aligned}
& u_{k}(i)= \begin{cases}1, & \text { if } i \in \Lambda(k) \\
0, & \text { otherwise }\end{cases} \\
& q_{k}(i)=\left\{\begin{array}{cl}
0, & \text { if } i \in F_{D}(k) \\
1, & \text { otherwise }
\end{array}\right.
\end{aligned}
$$

In defining the cost-function contribution $C_{x}(k, i)$, relative to the status of charunel $i$ in cell $k \in I(x)$, the first term takes into account the availability of channel $i$ in cell $k$, while the second term takes into account if channel $i$ belongs to the optimal set for cell $k$ (i.e., $F_{D}(k)$ ). This second term is weighted by a factor 2 to follow as much as possible the FCA channel distribution. Therefore, the overall cost function can be obtained as:

$$
C_{x}(i) \triangleq q_{x}(i)+\sum_{k \in I(x)}\left\{C_{x}(k, i)\right\}, \forall i \in \Lambda(x)
$$

where the terrn $q_{x}(i)$ is introduced in (8) because it is preferable to allocate in $x$ a channel $i \in F_{D}(x)$.

\section{B) Management of inter-beam handover requests}

Let us assume that an active MS is approaching the borders of its cell $x$ (=beam footprint) and is going into an adjacent cell $y$. In this new cell, the MS must be provided with a new channel to carry on the communication; subsequently, the channel used in the source cell must be released by the MS.

As a matter of fact, we assume that there is a certain degree of overlap among the footprints on the earth of adjacent beams. Within an overlap area an MS can receive the signal from two beams. This is a prerequisite to allow the Queuing of Handover requests $(\mathrm{QH})$. Let us assume that an MS sends a handover request as soon as it enters the overlap area between cell $x$ and cell $y$. The maximum waiting time for any handover request, 
$t_{\text {wmax }}$, can be derived as the time spent by an MS to cross the overlap area with a speed $V_{t r k}$. According to the proposed mobility model, we have assumed that the overlap area extension crossed by an MS is fixed and equal to $R / 5$. This is a conservative choice that considers a purely geometric overlap of circular coverage areas disposed according to a hexagonal layout, as explained in [5]. Therefore, $t_{\text {w }}$ max results in:

$$
t_{\text {xmax }}=\frac{\alpha T_{m}}{10}
$$

Of course, we must admit that the overlap area extension and then $t_{\text {wmax }}$ are random variable, that depend on several parameters, such as the direction of MS (relative to the spotbeam footprint) motion, the satellite antenna characteristics and the propagation conditions, but this aspect will not be addressed here where the deterministic value of $t_{\text {nmax }}$ given by (9) is used. An inter-beam handover request sent by an MS is served according to the following steps:

1) if it results $\Lambda(y) \neq \varnothing$, an arrival due to handover is served in cell $y$ and a call termination (see point $C$ of this Section) is performed in cell $x$,

2) if it results $\Lambda(y)=\varnothing$, the handover request is queued waiting for an available channel in cell $y$. In the meantime, the communication is served by the source cell. The queue service discipline is FIFO. A handover request leaves the queue owing to one of the three following reasons:

- the handover procedure is successful: the handover request is served, before the call is ended and the maximum queuing time has expired.

- The handover procedure has been useless: the associated call ends before the corresponding handover request is served and the maximum queuing time has expired.

- The handover procedure fails and the associated call is dropped: the handover has not been performed within $t_{\mathrm{w}, \mathrm{m} \text {. }}$ and the call is not ended before the maximum queuing time has expired.

\section{C) Management of call terminations}

In order to improve the performance of a DCA technique, we propose that whenever a call termination occurs in a cell $x$ (due either to the end of the call or to handover), the channel assignments in $x$ must be defined again to de-allocate in $x$ the channel at maximum cost. The cost-function to be used in this case is complementary to that defined at point $A$ to allocate new call arrivals. Then, if the most convenient channel to be deallocated is different from the channel released due to the call termination, a channel rearrangement (i.e., intra-beam handover) is required.

In the following, we will denote with DCA the technique previously described in [5], with DCA-QH the same allocation technique with queued handover requests [5] and with PPA-
DCA-QH a further refinement of the DCA-QH algorithm with the PPA policy is used to manage new call attempts.

\section{A FOLDED NETWORK OF CELLS}

The simulated network is parallelogram shaped; this is a common choice in the literature to study terrestrial cellular systems. However, in order to adapt this topology to the different characteristics of global MSSs, where the cellular network is three-dimensional, we define a folded network of cells to obtain that each cell has a complete belt of interfering cells. We denote with $N$ the number of cells per side in the cellular network. Each cell of the network has an identification number: $n=1,2,3, \ldots, N^{2}$ (see Fig. 1, where $N=7$ ). Let us denote with $\Pi$ the set of cells belonging to the simulated network: $\Pi=$ $\left\{1,2,3, \ldots, N^{2}\right\}$. Moreover, an oblique reference with the origin in the center of the cell No.1 is used, as shown in Fig. 1. If we normalize the distance among two adjacent cell centers, each cell center is denoted in this reference by a couple of integer numbers $(\xi, \eta)$. We assume that the belt of interfering cells of cell $x$, (i.e., $l(x)$ ) is formed by two tiers of adjacent cells. Then, the two orders of more external cells in the parallelogram shaped network have an incomplete belt of interfering cells and form the set of "border" cells, $B$. Consequently, we name "central" cells those cells belonging to the set $\Pi$ - B. Let us consider a generical cell $z \equiv\left(\xi_{z}, \eta_{z}\right)$ on the border of the network; a generical cell $k \in I(z)$ has the following coordinates: $k \equiv\left(\xi_{z}+a, \eta_{z}+b\right)$, where $a, b \in\{0$, $1,2,-1,-2\}$ and $|a|+|b| \neq 0$.

- If $k \in \Pi$, the interfering cell really exists;

- Otherwise, if $k \notin \Pi$, the interfering cell does not exist; then, $k$ becomes a dummy interfering cell of $z$ according to the following rule: the coordinates of the interfering cell $k$ $\equiv\left(\xi_{z}+a, \eta_{z}+b\right)$ are transformed into $k \equiv\left(\xi_{k}, \eta_{k}\right)$, where $\xi_{k}=\left(\xi_{z}+a\right) \bmod (N)$ and $\eta_{k}=\left(\eta_{z}+b\right) \bmod (N)$. Therefore, a cell on a border of the simulated network interferes with border cells on the other side of the network. In Fig. 1, the dummy interfering cells for border cell No. 48 are shown.

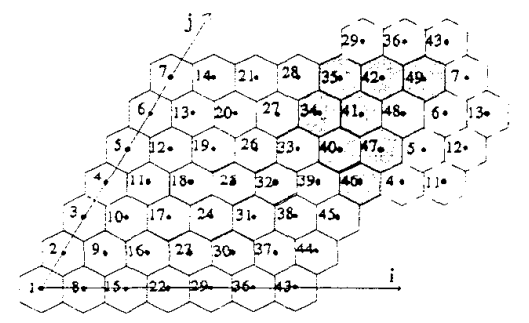

Fig. 1: An example of dummy interfering cells for the parallelogram shaped cellular network. 
When a folded network is used an MS with a call in progress, which goes out of a side of the network, re-enters the network from the other side, thus ideally reproducing the behavior of an infinite network. This is a significant advantage when the performance of a channel allocation technique is evaluated jointly with a mobility model for the users. Owing to the chosen topology of the simulated network (i.e., parallelogram shaped) and the size of the belt of interfering cells, if we use the costfunction defined in (8), the previous folded network is valid only for values of $N$ which are integer multiple of 7 . Only for these special values of $N$ the dummy interfering cells around a border cell have the nominal channel allocation that follows the same regular FCA pattern, $F_{D}(x)$, used for the network.

\section{SIMULATION RESULTS}

All the simulation results shown in this Section have been derived by assuming that:

- the call arrival process is Poisson independent from cell to cell with average call arrival rate per cell equal to $\lambda$,

- the call duration is exponentially distributed with average value equal to $T_{m}=3 \mathrm{~min}$

- the belt of interfering cells is formed by two tiers of cells,

- the simulated cellular network is parallelogram shaped with 7 cells per side (i.e., $N=7$ ),

- a number of 70 channels is available to the system,

- the IRIDIUM mobility case is considered $(\alpha \approx 0.32)$,

- the maximum queuing time is $t_{\text {mmax }} \approx 0.1 \mathrm{~min}$,

- an infinite queue capacity is assumed.

Let us denote with $P_{b 1}$ the blocking probability for new call arrival and with $P_{b 2}$ the inter-beam handover failure probability. In our simulations, we verified that the values of $P_{b 1}$ and $P_{b 2}$ as well as of $P_{n s}$ are uniform for all the cells of the simulated network. This is due to the folded network where all the cells have a complete belt of interfering cells. Another interesting result was the insensitivity of the blocking results to the network dimensions (i.e., to the value of $N$ which was assumed as an integer multiple of 7): e.g., the performance of the channel allocation techniques presented in this paper for $N=7$, was also evaluated in the case of $N=14$ and no significant difference was found. Therefore, the simulations carried out on a network of reduced dimensions give results that are also valid for networks of greater dimensions. This permits to speed-up the simulation runs.

In this paper, we have assumed that acceptable values of $P_{b 1}$ are of the order of 1-3\%, while the acceptable values of $P_{b 2}$ are equal to $10^{-4}$ (ITU-T recommendations). The comparison among DCA, DCA-QH and PPA-DCA-QH techniques in the IRIDIUM mobility case is shown in Figs. 2, 3 and 4 for $P_{b 1}, P_{b 2}$ and $P_{n s}$, respectively. We can see that the $P_{b 1}$ and $P_{b 2}$ performance of the DCA technique is unsatisfying, because of the high values of $P_{b 2}$ all over the traffic range under consideration (i.e., $P_{b 2}>10^{-4}$ ). A possible solution to decrease these values of $P_{b 2}$ is achieved by queuing handover requests. Then, we consider the DCA-QH technique that privileges the handover service at the expenses of an increased blocking for the new call arrivals. Finally, if we use the PPA-DCA-QH technique, it is possible to reduce this increase in $P_{b,}$ by accepting a negligible increase in $P_{b 2}$.

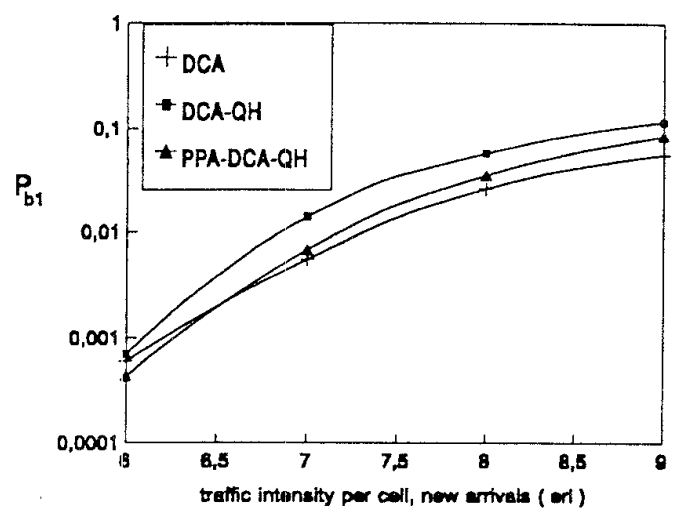

Fig. 2: $F_{b I}^{\prime}$ performance of DCA, DCA-QH and PPA-DCA-QH (IRIDIUM mobility case).

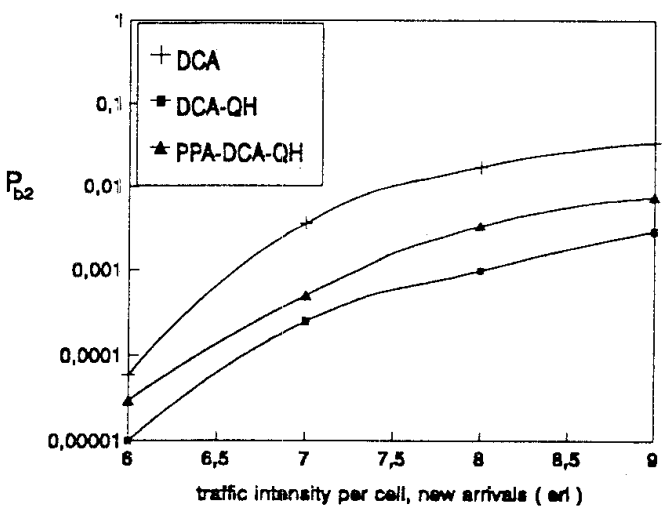

Fig. 3: $P_{1,2}$ performance of DCA, DCA-QH and PPA-DCA - QH (IRIDIUM mobility case).

The $P_{n s}$ performance for DCA, DCA-QH and PPA-DCA-QH techniques is presented in the curves of Fig. 4. Moreover, for the sake of completeness, the results for FCA and FCA-QH derived from [5] are also shown in this graph. We can conclude that the PPA-DCA-QH strategy achieves the best performance. 


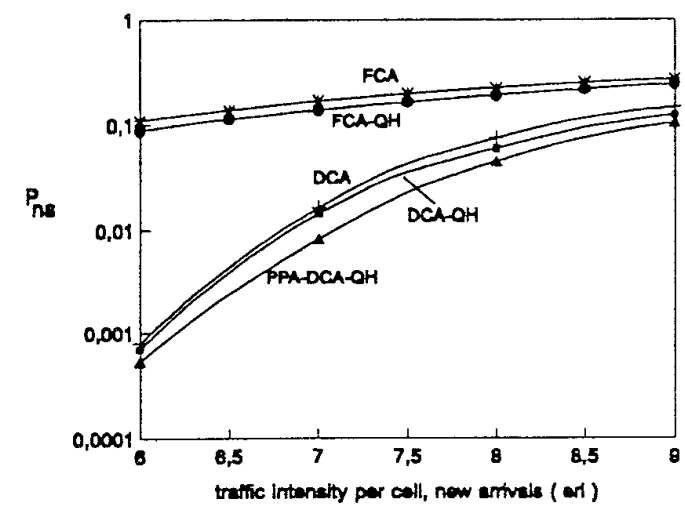

Fig. 4: $P_{n s}$ performance of DCA, DCA-QH and PPA-DCA-QH (IRIDIUM mobility case).

Another parameter to be evaluated in order to study the practical feasibility of the proposed channel allocation techniques is the signalling load carried out by the network. In particular, we consider only the signalling load supported by the network per served call to manage intra-beam (= "channel rearrangemen") and inter-beam handover procedures, $\sigma$. The events during the call lifetime that contribute to $\sigma$ and the relative contributions evaluated in terms of "number of signalling operations" are summarized in Table 1, taking into account the definition of the following parameter $\phi$ :

$$
\Phi \triangleq\left\{\begin{array}{l}
1, \text { if a channel rearrangement is required (10) } \\
0, \text { otherwise }
\end{array}\right.
$$

\begin{tabular}{|c|c|}
\hline EVENTS & CONTRIBUTIONS TO $\sigma$ \\
\hline new call arrival & $\phi$ \\
\hline handover & $2+\phi$ \\
\hline physical end & $\phi$ \\
\hline
\end{tabular}

Table 1: Contributions to $\sigma$ in correspondence with each event during call lifetime.

The question to be investigated here is whether the introduction of the PPA technique causes a significant increase in signalling load due to the channel rearrangements required to serve the new call arrivals which are otherwise blocked. To this end, we compare in Fig. 5 the $\sigma$ performance for DCA, DCA-QH and PPA-DCA-QH techniques. This figure highlights that with DCA $\sigma$ decreases when the traffic increases, because the handover failure probability, $P_{b 2}$, increases and, then, the average number of handover procedures per call decreases. Whereas different behaviors are obtained in the cases of DCA-QH and PPA-DCA$\mathrm{QH}$ techniques where the $P_{b 2}$ values are smaller than in the DCA case. The most interesting result is that PPA-DCA-QH technique does not increase $\sigma$ with respect to DCA-QH technique from low to medium traffic intensity per cell.

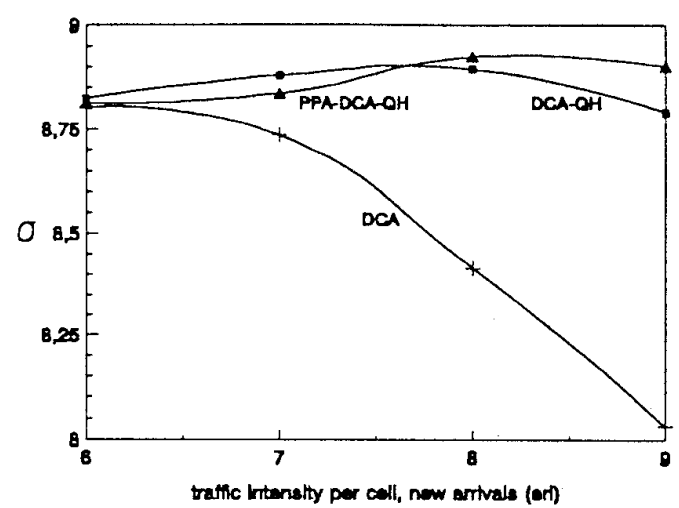

Fig. 5: Average signalling load per served call, $\sigma$, in the IRIDIUM case.

\section{CONCLUSIONS}

In this paper, an efficient DCA technique for applications in MSSs was proposed in order to cope with the increasing need of mobile satellite communications. The scenario envisaged was LEO-MSSs. A particular dynamic channel allocation technique based on the evaluation of a cost-function, namely DCA, was proposed. The queuing of handover requests was used to manage the user mobility. A performance improvement has been obtained in the case of the PPA-DCA-QH technique, where a new call arrival is accepted by the network even if there is not available channels by allowing a channel rearrangement in an interfering cell. Finally, it has been proven that the introduction of the PPA strategy does not increase the signalling load of the DCA-QH technique from low to medium traffic intensity per cell.

\section{BIBLIOGRAPHIC REFERENCES}

[1] J. F. Huber, "System Alternatives - Satellites and Network Aspects", First European Workshop on Mobile/Personal Satcons, Panel Discussion B, Frascati, Italy, October 14, 1994.

[2] E. Del Re, "Satellite System Integrated with the Terrestrial Cellular Network for Mobile Communications," ESA Tech. Rep., ESA STR-228, August 1989.

[3] A. Ganz, Y. Gong, Bo Li, "Performance Study of Low EarthOrbit Satellite Systems," IEEE Trans. on Comm., Vol. 42, n. 2/3/4, pp. 1866 - 1871, February/March/April 1994.

[4] Yi-Bing Lin, Seshadri Mohan, and Antony Noerpel, "PCS Channel Assignment Strategies for Hand-off and Initial Access," IEEE Personal Communicalions, pp. 47 - 56, Third Quarter 1994

[5] E. Del Re, R. Fantacci, G. Giambene, "Efficient Dynamic Channel Allocation Techniques with Handover Queuing for Mobile Satellite Networks," IEEE Journal on Selecled Areas in Commun., Vol. 13, n. 2, pp. 397-405, February 1995.

[6] E. Del Re, R. Fantacci, G. Giambene, "Performance Analysis of a Dynamic Channel Allocation Technique for Satellite Mobile Cellular Networks," International Journal of Satellite Communications, Vol. 12, pp. 25-32, January/February 1994.

[7] L. J. Cimini, G. J. Foschini, Chih-Lin I, and Z. Miljanic, "Call Blocking Performance for Dynamic Channel Allocation in Microcells," IEEE Trans. on Comm., Vol. 42, n. 8, pp. 2600. 2607, August 1994 